\title{
Prolonged left ventricular dysfunction occurs in patients with coronary artery disease after both dobutamine and exercise induced myocardial ischaemia
}

\author{
E Barnes, C S R Baker, D P Dutka, O Rimoldi, C A Rinaldi, P Nihoyannopoulos, \\ P G Camici, R J C Hall
}

\begin{abstract}
Objective-To determine whether pharmacological stress leads to prolonged but reversible left ventricular dysfunction in patients with coronary artery disease, similar to that seen after exercise. Design-A randomised crossover study of recovery time of systolic and diastolic left ventricular function after exercise and dobutamine induced ischaemia.
\end{abstract}

Subjects-10 patients with stable angina, angiographically proven coronary artery disease, and normal left ventricular function.

Interventions-Treadmill exercise and dobutamine stress were performed on different days. Quantitative assessment of systolic and diastolic left ventricular function was performed using transthoracic echocardiography at baseline and at regular intervals after each test.

Results-Both forms of stress led to prolonged but reversible systolic and diastolic dysfunction. There was no difference in the maximum double product $(p=0.53)$ or ST depression $(p=0.63)$ with either form of stress. After exercise, ejection fraction was reduced at 15 and 30 minutes compared with baseline (mean (SEM), -5.6 (1.5)\%, p < 0.05; and -6.1 (2.2)\%, p < 0.01), and at 30 and 45 minutes after dobutamine $(-10.8(1.8) \%$ and $-5.5(1.8) \%$, both $\mathrm{p}<0.01)$. Regional analysis showed a reduction in the worst affected segment 15 and 30 minutes after exercise $(-27.9(7.2) \%$ and $-28.6(5.7) \%$, both $\mathrm{p}<0.01)$, and at 30 minutes after dobutamine $(-32$ $(5.3) \%, p<0.01)$. The isovolumic relaxation period was prolonged 45 minutes after each form of stress $(p<0.05)$.

Conclusions-In patients with coronary artery disease, dobutamine induced ischaemia results in prolonged reversible left ventricular dysfunction, presumed to be myocardial stunning, similar to that seen after exercise. Dobutamine induced ischaemia could therefore be used to study the pathophysiology of this phenomenon further in patients with coronary artery disease.

(Heart 2000;83:283-289)

Keywords: myocardial stunning; echocardiography; exercise; dobutamine

Myocardial stunning is the reversible left ventricular dysfunction that persists after an episode of ischaemia despite the restoration of myocardial blood flow. ${ }^{12}$ It was initially described in $1975 .^{3}$ Subsequently it has been confirmed in the human in several clinical settings of transient ischaemia and reperfusion. ${ }^{4}$ The ventricular dysfunction during myocardial stunning recovers slowly after the inducing episode and its severity is dependent on the intensity and duration of the bout of ischaemia. ${ }^{5}$ Abnormalities in systolic function can last for up to one hour, whereas diastolic abnormalities can last for several hours after an episode of angina. Myocardial hibernation is the chronic left ventricular dysfunction seen in patients with coronary artery disease that is reversible after revascularisation. ${ }^{7}$ Initially believed to be caused by chronic reduction in myocardial blood flow leading to a chronic downregulation of left ventricular function, ${ }^{8}$ recent studies have shown that resting myocardial blood flow to hibernating myocardium is within normal limits in most patients. ${ }^{9}$ This has led to the hypothesis, first proposed by Vanoverschelde et $a l^{10}$ and supported by animal experiments, ${ }^{11}$ that hibernation may result from repeated episodes of ischaemia followed by reperfusion and postischaemic stunning. Prolonged repetitive stunning could culminate in chronic dysfunction and be the trigger for hibernation. ${ }^{12}$

Exercise induced stunning was first demonstrated in a canine model, ${ }^{13}$ and has been shown to occur in patients with coronary artery disease. ${ }^{14-17}$ Pharmacological stress generated by dobutamine has been extensively studied and is widely used to induce and detect myocardial ischaemia in patients with suspected coronary artery disease. ${ }^{18}$ We have recently shown that there is delayed recovery of myocardial function following dobutamine induced ischaemia and its duration is related to the extent of ischaemia. ${ }^{19}$ This delayed recovery of regional wall motion abnormality may represent myocardial stunning.

There has been much animal work on the pathophysiology of myocardial stunning. In humans this has been hindered by the need for simultaneous measurements of myocardial blood flow and function. No study to date has been able to assess myocardial blood flow quantitatively during stunning in patients with coronary artery disease. Positron emission 
tomography allows the quantitative assessment of myocardial blood flow and metabolism. ${ }^{90}$ Subject movement during data acquisition is one of the most important sources of error with this technique, and so it cannot be used to investigate exercise induced ischaemia. If dobutamine induced ischaemia produces prolonged but reversible postischaemic left ventricular dysfunction as seen after exercise induced ischaemia, subject movement would be eliminated and this would allow further studies on the pathophysiology of this condition. Our aim in this study was therefore to formally compare the effect of exercise and dobutamine induced ischaemia on the recovery of left ventricular function after each form of stress.

\section{Methods}

\section{PATIENT SELECTION}

We studied 11 patients (nine men, two women; mean age 57.5 years, range 48 to 67 ) with chronic stable angina pectoris, normal resting left ventricular function as assessed by both left ventriculography and echocardiography, and significant coronary artery disease $(\geqslant 70 \%$ stenosis in at least two major epicardial coronary arteries at coronary angiography $<4$ months before randomisation). All patients were taking aspirin, and one patient (patient 9) was taking an angiotensin converting enzyme inhibitor (table 1). Patients were excluded if there was a history of myocardial infarction or unstable angina within six months of recruitment and if it was not possible to obtain echocardiograms of sufficient quality for quantitative analysis.

STUDY PROTOCOL

Patients performed an exercise test and underwent pharmacological stress on different days. They were randomised to the mode of stress applied first. $\beta$ Blockers and calcium antagonists were withdrawn 48 hours before each study and other antianginal treatment was withdrawn on the day of the study. Patients were asked to avoid activities that might precipitate angina for 12 hours before each study and were excluded if they had suffered angina or used glyceryl trinitrate within four hours of the test. The study protocol was approved by the research ethics committee of the Hammersmith Hospital, Imperial College School of Medicine. All patients gave full written informed consent.
EXERCISE TESTING

Exercise testing was performed using a modified Bruce protocol. Three ECG leads (II, V2, and V5) were continuously monitored during exercise and recovery. Blood pressure and a 12 lead ECG were recorded every three minutes during exercise and every two minutes during recovery until resolution of ECG changes and a return of blood pressure and heart rate to baseline. Patients exercised until they experienced chest pain, were limited by other symptoms, or reached their target heart rate. Exercise was also discontinued if there was a drop in systolic blood pressure by $>10 \mathrm{~mm} \mathrm{Hg}$ or significant arrhythmias were induced.

\section{DOBUTAMINE STRESS}

Dobutamine was given in incremental doses using an infusion pump (starting at $5 \mu \mathrm{g} / \mathrm{kg} / \mathrm{min}$ and increasing by $5 \mu \mathrm{g} / \mathrm{kg} / \mathrm{min}$ at three minute intervals to a maximum of $40 \mu \mathrm{g} / \mathrm{kg} / \mathrm{min}$ if tolerated). Patients not achieving $90 \%$ of their maximum predicted heart rate were given 0.6 $\mathrm{mg}$ of atropine intravenously, and this was repeated once after five minutes if necessary. Atropine was not given if the patient had signs or symptoms of ischaemia. Three ECG leads (II, V2, and V5) were monitored continuously during pharmacological stress and recovery. Blood pressure and a 12 lead ECG were recorded every three minutes during stress and every two minutes during recovery until resolution of ECG changes and return of blood pressure and heart rate to baseline. The end points were similar to those with the exercise protocol.

\section{ECHOCARDIOGRAPHY}

Cross sectional echocardiography was performed with the patient in the left recumbent position using commercially available equipment (HDI 3000 and 5000, ATL Ltd, Bothell, Washington, USA) with a P3-2 broadband phased array transducer. All studies were performed by the same investigator (EB). Images were recorded at rest and then at 15 minute intervals for one hour, and then at two hours and three hours after both forms of stress. To ensure reproducibility, the position of the patient on the couch was recorded at baseline and used for subsequent studies. To minimise beat to beat variability, all recordings were made in gently held expiration. ${ }^{21}$ Studies were recorded onto super VHS videotape for off line

Table 1 Demographic data

\begin{tabular}{lllllll}
\hline Patient & $\begin{array}{l}\text { Age } \\
\text { (years) }\end{array}$ & $\begin{array}{l}\text { Height } \\
(\mathrm{cm})\end{array}$ & $\begin{array}{l}\text { Weight } \\
(\mathrm{kg})\end{array}$ & Primary diagnosis & Drugs & Coronary disease* \\
\hline 1 & 52 & 155 & 80 & Angina pectoris & Atenolol, ISMN & LAD, RCA \\
2 & 62 & 175 & 83 & Angina pectoris & Atenolol, ISMN & LAD, RCA \\
3 & 57 & 181 & 89 & Angina pectoris & Atenolol & LAD, OM, RCA \\
4 & 48 & 173 & 97 & Angina pectoris & Atenolol & LAD, Cx, RCA \\
5 & 65 & 165 & 77 & Angina pectoris & Amlodipine, ISMN & LAD, Cx, RCA, Diag \\
6 & 51 & 185 & 100 & Angina pectoris & Metoprolol & LAD, Cx, OM, RCA \\
7 & 66 & 158 & 65 & Angina pectoris & Atenolol, amlodipine & Diag, OM, RCA \\
8 & 49 & 165 & 65 & Angina pectoris & Bisoprolol, ISMN & LAD, Cx \\
9 & 58 & 174 & 89 & Angina pectoris & Lisinopril & LAD, OM \\
10 & 67 & 156 & 69 & Angina pectoris & Amlodipine & LAD, Diag, RCA \\
\hline
\end{tabular}

${ }^{\star}$ More than $70 \%$ stenosis.

$\mathrm{Cx}$, circumflex coronary artery; Diag, diagonal branch of left anterior descending coronary artery; ISMN, isosorbide mononitrate; LAD, left anterior descending coronary artery; OM, obtuse marginal branch of circumflex coronary artery; RCA, right coronary artery. 
analysis. Left ventricular contractile function was assessed in the apical two chamber $(\mathrm{AP} 2 \mathrm{CH})$ and apical four chamber $(\mathrm{AP} 4 \mathrm{CH})$ views, as described in the guidelines from the American Society of Echocardiography. ${ }^{22}$ Pulse wave Doppler tracings of the left ventricular diastolic inflow, at the tips of the mitral valve, were recorded. The isovolumic relaxation period was measured using pulse wave Doppler tracings recorded from simultaneous recordings of the left ventricular diastolic inflow and the end of the systolic outflow as described previously. ${ }^{23}$

ECHOCARDIOGRAPHIC ANALYSIS

The videotaped images were analysed using an off line PC based digitising program (Thoraxcenter, Erasmus University, Rotterdam, Netherlands). ${ }^{21}$ Three consecutive beats (excluding extrasystolic and postextrasystolic beats) were analysed for each time point in the $\mathrm{AP} 2 \mathrm{CH}$ and $\mathrm{AP} 4 \mathrm{CH}$ views. Endocardial borders (excluding papillary muscles) were traced at end diastole, timed as the closure of the mitral valve leaflets, and at end systole, defined as the point of maximum inward excursion of the endocardial contour. The centreline method was used to assess regional left ventricular function in which the computer superimposes the end diastolic and end systolic endocardial tracings and calculates a centreline between the two. The deviation from the centreline of 100 chords around the left ventricular circumference is calculated after correction for the end diastolic circumference and expressed as a percentage shortening fraction. Each apical view of the left ventricle was divided into six segments and the shortening fraction of the chords in each segment was averaged so that 12 values were obtained (six $\mathrm{AP} 4 \mathrm{CH}$ and six $\mathrm{AP} 2 \mathrm{CH}$ ). The shortening fraction for all 12 segments (SFmean) and in the segments that developed the worst impairment after stress (SFworst) was calculated. Left ventricular volumes at end diastole and end systole were calculated using the biplane disk method, and global ejection fraction was derived as previously described. ${ }^{24}$ The coefficient of repeatability for this method was $2.6 \%$ as assessed by the method of Bland and Altman. ${ }^{25}$ The isovolumic relaxation period was used as an index of diastolic function. Analysis was performed using a digitising tablet and tracing the pulse wave Doppler signal (four consecutive beats) for each time point.

STATISTICAL ANALYSIS

The primary end points were based on a comparison of the effects of exercise stress and pharmacological stress on the echocardiographic indices of left ventricular function (ejection fraction, SFworst, SFmean, and isovolumic relaxation period). Secondary end points were maximum double product and maximum ST segment depression. Data (mean $(\mathrm{SEM}))$ are presented as overall percentage change from baseline, except isovolumic relaxation period (ms). All echocardiographic data met the assumption of homogeneity of variances required for using analysis of vari- ance (ANOVA). Within each group, recovery of echocardiographic variables and ST segment depression was tested for significance by one way ANOVA, with a design for repeated measures. When the overall analysis showed a significant trend, differences in the different time points versus baseline were tested by use of Dunnett's test to correct for multiple comparisons. A value of $p<0.05$ was considered significant. Heart rate and blood pressure differences were assessed using a paired Student's $t$ test.

\section{Results}

Data are available for 10 of the 11 patients. One subject (male) was excluded because he developed severe myocardial ischaemia during the dobutamine infusion, and echocardiographic data acquisition was not possible. Two patients required atropine $0.6 \mathrm{mg}$ (patients 5 and 6 ) in addition to dobutamine to attain maximal heart rate. Diastolic data are available for nine subjects. Doppler acquisition was not possible in one subject (patient 7).

\section{PRIMARY OUTCOMES}

With both types of stress there was prolonged but reversible systolic and diastolic left ventricular dysfunction. The overall duration of systolic and diastolic dysfunction was greater after dobutamine than after exercise induced ischaemia.

Effects of stress on global left ventricular function

Treadmill exercise led to a reduction in ejection fraction 15 and 30 minutes after stress (mean (SEM), $-5.6 \quad(1.5) \% \quad \mathrm{p}<0.05$ and -6.1 $(2.2) \%, p<0.01$ for both) (fig 1). After dobutamine the ejection fraction was reduced 30 and 45 minutes after stress $(-10.8(1.8) \%$, $\mathrm{p}<0.01$; and $-5.5(1.8) \%, \mathrm{p}<0.05$, respectively), but not after 15 minutes. Data at other time points were not significantly different from baseline. Exercise led to a decrease in global ejection fraction in eight of the 10 patients. In the other two patients, dobutamine stress led to a reduction in ejection fraction not

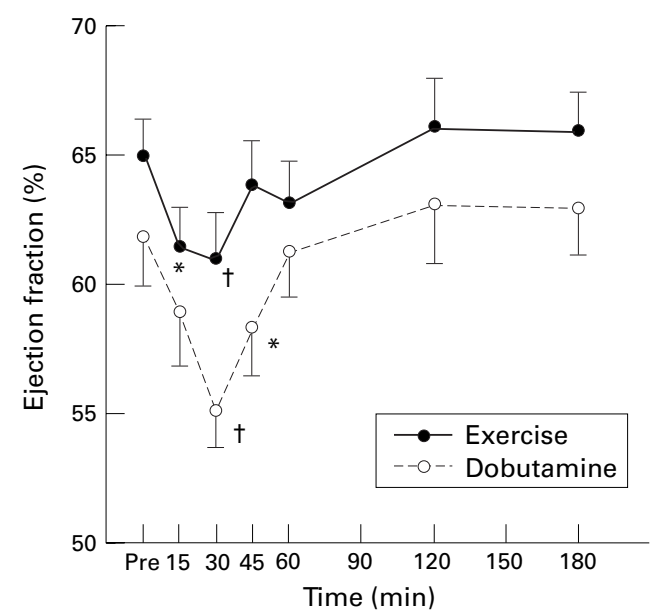

Figure 1 Effect of exercise and dobutamine induced stress on mean global ejection fraction. Error bars $=S E M .{ }^{*} p<$ $0.05 ; \dagger p<0.01$. 


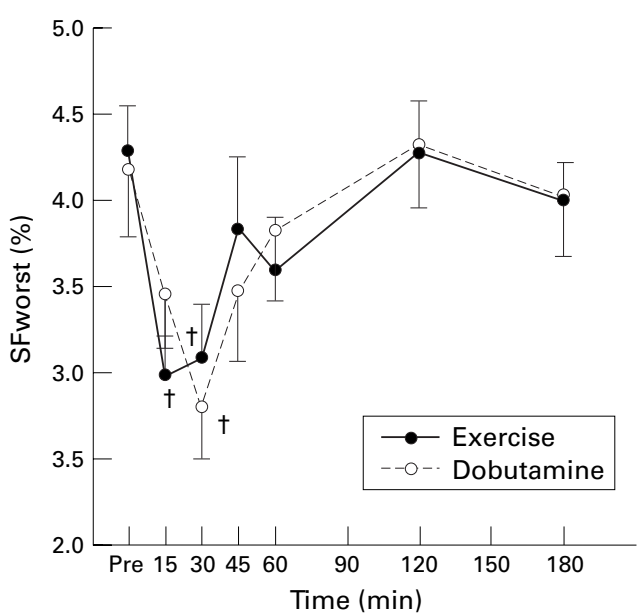

Figure 2 Effect of exercise and dobutamine induced stress on mean SFworst (shortening fraction in the segments that developed the worst impairment after stress). Error bars $=$ SEM. $\uparrow p<0.01$.

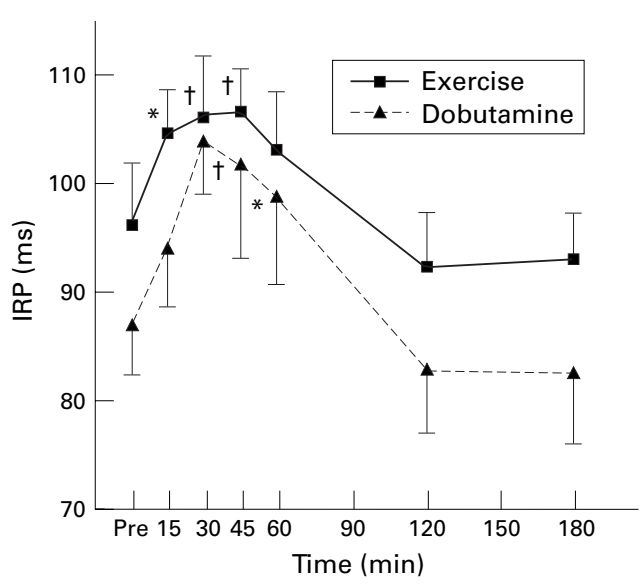

Figure 3 Effect of exercise and dobutamine induced stress on mean isovolumic relaxation period. Error bars $=S E M$. ${ }^{*} p<0.05 ; \dagger p<0.01$.

seen after exercise. One patient had a reduction in ejection fraction after exercise, but not after dobutamine stress.

Regional left ventricular function

Regional analysis showed a reduction in SFworst 15 and 30 minutes after exercise $(-27.9(7.2) \%$ and $-28.6(5.7) \%, \mathrm{p}<0.01$ for both). After dobutamine, there was a reduction in SFworst at 30 minutes $(-32 \% \quad$ (5.3), $\mathrm{p}<0.01$ ) (fig 2).

\section{SFmean}

The SFmean was reduced 15 and 30 minutes after exercise $(-8.4(1.8) \%, \mathrm{p}<0.05$; and $-10.3(4.1) \%, \mathrm{p}<0.01$, respectively), at 30 and 45 minutes after dobutamine $(-15.7$ (2.3) \% and $-10.4(2.4) \%, \mathrm{p}<0.01$ for both), and 60 minutes after dobutamine $(-8.9$ $(1.8) \%, p<0.05)$.

Isovolumic relaxation period

The mean isovolumic relaxation period at baseline was 96 (5.7) and 87 (4.5) ms for exercise and dobutamine, respectively. It was significantly prolonged at 15,30 , and 45
Table 2 Exercise and dobutamine stress results

\begin{tabular}{lll}
\hline & Exercise & $\begin{array}{l}\text { Dobutamine } \\
\text { stress }\end{array}$ \\
\hline Resting heart rate (beats/min) & $77(3.0)$ & $74(3.3)$ \\
Peak heart rate (beats/min) & $127(6.2)$ & $132(3.4)$ \\
Resting systolic BP (mm Hg) & $124(5.4)$ & $130(5.3)$ \\
Resting diastolic BP (mm Hg) & $82(2.8)$ & $76(3.5)$ \\
Peak systolic BP (mm Hg) & $166(5.8)$ & $144(7.3)$ \\
Peak diastolic BP (mm Hg) & $97(10.1)$ & $69(5.4)^{\star}$ \\
Maximum ST depression (mm) & $1.54(0.25)$ & $1.20(0.24)$ \\
Rate-pressure product & $21547(1585)$ & $19040(1069)$ \\
Duration of stress (s) & $552(89)$ & $916(80) \dagger$ \\
\hline
\end{tabular}

Values are mean $(\mathrm{SEM})$.

${ }^{\star} \mathrm{p}<0.05 ; \mathrm{p}=0.005$.

$\mathrm{BP}$, blood pressure.

minutes after exercise compared with baseline (104.4 (4.2) ms, p < 0.05; 106 (5.4) ms, $\mathrm{p}<0.01$; and 106.5 (4.0) ms, $\mathrm{p}<0.01$, respectively) (fig 3). After dobutamine the isovolumic relaxation period was significantly prolonged compared with baseline after 30 and 45 minutes (103.9 (4.8) $\mathrm{ms}$ and 101.6 (8.5) $\mathrm{ms}$, respectively; both $\mathrm{p}<0.05)$.

\section{SECONDARY OUTCOMES}

These are presented in table 2. There was no significant difference in maximum double product between exercise and dobutamine (21 547 (1585) v 19040 (1069), $\mathrm{p}=0.53$ ). Maximum ST depression was $1.55(0.24) \mathrm{mm}$ for exercise and $1.20(0.24) \mathrm{mm}$ for dobutamine $(p=0.63)$. ST segment depression took longer to recover after dobutamine than after exercise. Following exercise there was significant ST segment depression at five minutes into recovery but not at 10 minutes, whereas after dobutamine, ST segment depression was still significantly reduced at 10 minutes (fig 4). At 15 minutes, when the first echocardiographic acquisition took place, ST segment depression was not significantly different from baseline with either form of stress. There was no difference in heart rate either before or after different forms of stress.

We observed an increase in both the peak systolic and diastolic blood pressure in the exercise group compared with the pharmacological stress group (table 2). The difference in diastolic blood pressure was significant $(p=0.04)$. One patient (patient 7) had a fall in systolic and diastolic blood pressure at peak stress with dobutamine (120/70 resting, 96/49 peak).

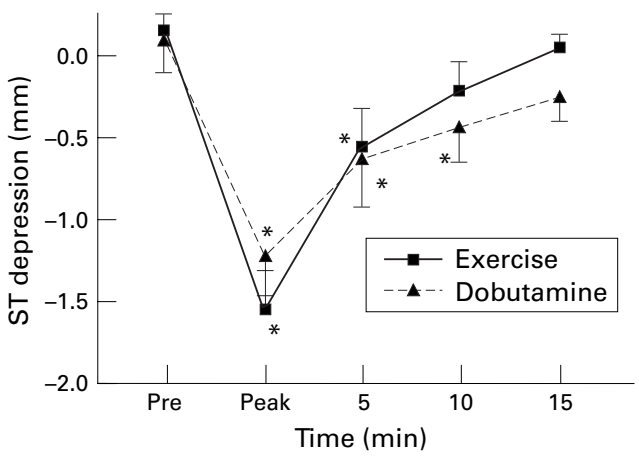

Figure 4 ST segment depression after exercise and dobutamine induced ischaemia. ${ }^{*} p<0.05$. 


\section{Discussion}

This study shows that ischaemia induced by pharmacological stress using dobutamine leads to prolonged, but reversible systolic and diastolic left ventricular dysfunction, probably caused by myocardial stunning. This occurs at a time when there has been resolution of ST segment depression. Moreover exercise and dobutamine stress have different effects on the recovery of left ventricular function, which is more prolonged after dobutamine induced ischaemia. To our knowledge this is the first study to have shown this in the human.

Myocardial stunning was first described by Heyndrickx and coworkers in 1975 in a canine model of transient coronary occlusion. ${ }^{3}$ It has now been confirmed that it occurs in the human in the clinical settings of transient ischaemia and reperfusion. ${ }^{4}$ These include acute coronary syndromes (such as myocardial infarction and unstable angina), coronary angioplasty, coronary artery bypass grafting, coronary spasm, and exercise induced ischaemia. Exercise induced stunning was first described by Homans et al in dogs. ${ }^{13}$ There have been similar findings in patients with chronic coronary artery disease, in whom exercise echocardiography showed wall motion abnormalities with exercise that persisted for up to 30 minutes, ${ }^{15}$ although that study did not equate these changes to stunning. Other investigators have confirmed these findings. ${ }^{6}{ }^{16}{ }^{17}$ In a recent study, Ambrosio et al demonstrated reversible left ventricular dysfunction in the presence of normal myocardial blood flow, assessed using MIBI after exercise induced ischaemia - the crux of myocardial stunning. ${ }^{14}$

Dobutamine stress is widely used as an alternative to exercise for the diagnosis of coronary artery disease. It has been shown to lead to ischaemia and myocardial stunning in an open chested canine model with an acute coronary stenosis. ${ }^{26}$ Tsoukas et al have studied the recovery of systolic left ventricular function after dobutamine stress. $^{19}$ They showed that the recovery of regional wall motion abnormalities induced by dobutamine occurs later than the resolution of symptoms and ECG changes. In patients with two vessel disease, $68 \%$ had persistent regional wall motion abnormality at 10 minutes and $23 \%$ at 15 minutes. In patients with three vessel disease, all regions recovered by 25 minutes, but $29 \%$ had persistent changes at 20 minutes. They concluded that the duration of regional wall motion abnormality was related to the severity of the coronary artery disease and that the prolonged dysfunction may reflect myocardial stunning. Our observations are similar, although the recovery time after dobutamine in our study was longer. The analysis package that we used may be more sensitive than the visual analysis of wall motion score used by Tsoukas et al, and this may have accounted for the differences. The time scale for recovery after exercise in our study is in agreement with other published data. ${ }^{1424}$ In the study by Ambrosio et $a l^{8}{ }^{8}$ there were simultaneous flow data showing that myocardial blood flow was normal at the time of left ventricular dysfunction, thereby confirming these changes to be caused by myocardial stunning.

The time course of recovery of systolic left ventricular dysfunction after dobutamine differed from exercise in two respects. First, the changes persisted for longer; thus 45 minutes after dobutamine stress, global left ventricular function remained reduced. This was not the case after exercise. The SFmean was still significantly reduced 60 minutes after dobutamine, but had resolved within 45 minutes after exercise. Second, the changes after exercise occurred more rapidly: 15 minutes after exercise, left ventricular function was significantly impaired, but not after dobutamine. The findings with exercise are in accordance with other published studies. ${ }^{14}{ }^{24}$ The severity and duration of myocardial stunning after exercise is related to the severity and the duration of the episode of ischaemia, although the former is the more important. ${ }^{6}$ Without data on flow, it is not possible to establish the severity of ischaemia with either form of stress. This is also the case if one uses non-invasive indices of ischaemia such as ST depression. It is well established that the sensitivity of ECG changes during dobutamine induced ischaemia is poor. Shaheen et al reported a sensitivity of only $52 \%$ in patients with angiographically proven coronary artery disease. ${ }^{28}$ Mazeika et al compared the diagnostic accuracy of ECG changes with that of dobutamine stress echocardiography for the detection of $>70 \%$ stenosis in 54 patients referred for coronary angiography. ${ }^{29}$ They reported a low sensitivity and negative predictive accuracy of ECG alone $(47 \%$ and $34 \%$, respectively). Daoud et al reported a similar sensitivity of $47 \%$. In these studies the specificity and positive predictive accuracy was far greater, ranging from $53 \%$ to $71 \%$ and $73 \%$ to $81 \%$, respectively. ${ }^{30}$ There are also data suggesting that ischaemia occurs at a lower level of external cardiac work during dobutamine compared with exercise stress. ${ }^{31}$ It may therefore be that for the same rate-pressure product and ST segment depression the ischaemic burden with dobutamine is greater. It is thus possible that these patients became more ischaemic with dobutamine stress despite a trend to less ST segment depression. This would be in contrast to the study by Rallidis et $a l,^{32}$ who compared dobutamine and treadmill exercise echocardiography in inducing ischaemia in patients with coronary artery disease. That study showed that treadmill exercise induced a greater ischaemic burden than dobutamineatropine infusion, as assessed by the occurrence of new regional motion abnormalities at peak stress. This was not an end point in our study.

It is also possible that the prolongation in left ventricular dysfunction seen after dobutamine may be the result of a prolonged period of stress and a longer period of ischaemia. The average exercise time was 552 (89), seconds compared with an infusion time of dobutamine of 916 (80) seconds $(p=0.005)$. It may therefore be that patients were stressed for a longer time, leading to more prolonged left ventricular 
dysfunction. This is in agreement with the study by Homans et al who studied chronically instrumented dogs with a circumflex stenosis. ${ }^{6}$ The animals were subjected to exercise stress of a degree adequate to increase coronary flow $50 \%$ above baseline. Animals exercised for five minutes, and on another occasion for 10 minutes. The investigators showed that the severity of exercise induced stunning was similar for both groups but that the recovery was slower in the animals after 10 minutes of exercise.

The differences in the early time course in recovery of both systolic and diastolic function may be explained by the time taken for the resolution of ischaemia after each form of stress. Although ST segment analysis has a poor sensitivity in detecting ischaemia during dobutamine infusion, the specificity and positive predictive accuracies are superior. There was no significant difference in the ST segment depression compared with baseline at 15 minutes, when the first echocardiographic data after stress were collected. Despite this, the recovery of ST segment depression took longer after dobutamine. Ten minutes after the infusion of dobutamine was stopped, ST segments were still depressed compared with baseline, whereas after exercise there was no significant difference (fig 4). The half life of dobutamine is two minutes and therefore its effects may take longer to resolve than that of exercise stress. Allen et al have shown that after exercise induced stunning in patients with severe coronary artery disease, partial recovery is observed during the first 15 minutes, with a subsequent deterioration in function that is maintained to 45 minutes. ${ }^{33}$ This finding was substantiated by Rinaldi et $a l^{34}$ who also showed this biphasic response after exercise, although the improvement in function was earlier than that demonstrated by Allen et al. There are no data on the resolution of baseline myocardial blood flow after dobutamine, although it has been investigated after exercise. ${ }^{14203536}$ Indeed, Camici et al found that myocardial blood flow had recovered by $5-14$ minutes. $^{20}$

In our study there was a trend for the time course of recovery of diastolic function to be prolonged after dobutamine, but this was not statistically significant. The isovolumic relaxation period was significantly increased $45 \mathrm{~min}$ utes after both forms of stress. Fragasso et al found sustained diastolic dysfunction after exercise in patients with coronary artery disease. ${ }^{37}$ In their study the peak filling rate, measured by radionuclide ventriculography, remained depressed for up to 48 hours after stress. The extremely prolonged abnormalities in diastolic function in Fragasso's study may well have been caused by further episodes of ischaemia after the original exercise test, as no ST segment analysis was undertaken. This may have led to further stunning before reassessment at two days. Rinaldi et al, who examined diastolic function using the isovolumic relaxation period, had findings similar to ours. ${ }^{24}$
CONCLUSIONS

We have shown that in patients with coronary artery disease dobutamine induced ischaemia leads to prolonged but reversible systolic and diastolic dysfunction despite resolution of ST segment depression. The persistence of left ventricular dysfunction is greater after dobutamine than after exercise stress. It has previously been shown that when these changes occur after exercise induced ischaemia they are caused by myocardial stunning; we believe that this is also the case following dobutamine. Further studies with simultaneous measurements of myocardial blood flow are required to substantiate this.

EB is supported by a Wellcome Research Training Fellowship.

1 Braunwald E, Kloner RA. The stunned myocardium: prolonged, postischemic ventricular dysfunction. Circulation 1982;66:1146-9.

2 Bolli R. Mechanism of myocardial "stunning". Circulation 1990;82:723-38.

3 Heyndrickx GR, Millard RW, McRitchie RJ, et al. Regional myocardial functional and electrophysiological alterations after brief coronary artery occlusion in conscious dogs. $\mathcal{F}$ Clin Invest 1975;56:978-85.

4 Bolli R. Basic and clinical aspects of myocardial stunning. Prog Cardiovasc Dis 1998;40:477-516.

5 Bolli R, Zhu WX, Thornby JI, et al. Time course and determinants of recovery of function after reversible ischemia in conscious dogs. Am f Physiol 1988;254:H102-14.

6 Homans DC, Laxson DD, Sublett E, et al. Effect of exercise intensity and duration on regional function during and afte exercise-induced ischemia. Circulation 1991;83:2029-37.

7 Wijns W, Vatner SF, Camici PG. Hibernating myocardium. N Engl f Med 1998;339:173-81.

8 Rahimtoola SH. The hibernating myocardium. Am Heart 7 1989;117:211-21.

9 Camici PG, Rimoldi O. Resting myocardial blood flow in patients with hibernating myocardium quantified by positron emission tomography. Basic Res Cardiol 1997; 92(suppl 2):6-8.

10 Vanoverschelde JJ, Wijns W, Depre C, et al. Mechanisms of chronic regional postischaemic dysfunction in humans. New insights from the study of noninfarcted collateraldependent myocardium. Circulation 1993;87:1513-23.

11 Shen YT, Vatner SF. Mechanism of impaired myocardial function during progressive coronary stenosis in conscious pigs. Hibernation versus stunning? Circ Res 1995;76:479-88.

12 Kloner RA, Bolli R, Marban E, et al. Medical and cellular implications of stunning, hibernation, and preconditioning: an NHLBI workshop. Circulation 1998;97:1848-67.

13 Homans DC, Sublett E, Dai XZ, et al. Persistence of regional left ventricular dysfunction after exercise-induced myocardial ischemia. $\mathcal{F}$ Clin Invest 1986;77:66-73.

14 Ambrosio G, Betocchi S, Pace L, et al. Prolonged impairment of regional contractile function after resolution of exercise-induced angina. Evidence of myocardial stunning in patients with coronary artery disease. Circulation 1996;94:2455-64

15 Robertson WS, Feigenbaum H, Armstrong WF, et al. Exercise echocardiography: a clinically practical addition in the evaluation of coronary artery disease. $\mathcal{F} \mathrm{Am}$ Coll Cardiol 1983;2:1085-91.

16 Scognamiglio R, Ponchia A, Fasoli G, et al. Exerciseinduced left ventricular dysfunction in coronary heart disease. A model for studying the stunned myocardium in man. Eur Heart f 1991;12(suppl G):16-19.

17 Kloner RA, Allen J, Cox TA, et al. Stunned left ventricular myocardium after exercise treadmill testing in coronary artery disease. Am f Cardiol 1991;68:329-34.

18 Senior R, Kenny A, Nihoyannopoulos P. Stress echocardiography for assessing myocardial ischaemia and viable myocardium. Heart 1997;78(suppl 1):12-18.

19 Tsoukas A, Ikonomidis I, Cokkinos P, et al. Significance of persistent left ventricular dysfunction during recovery after dobutamine stress echocardiography. $7 \mathrm{Am}$ Coll Cardiol 1997;30:621-6.

20 Camici P, Araujo LI, Spinks T, et al. Increased uptake of $18 \mathrm{~F}-$ fluorodeoxyglucose in postischemic myocardium of patients with exercise-induced angina. Circulation 1986;74:81-8.

21 Assmann PE, Slager CJ, van-der-Borden SG, et al. Quantitative echocardiographic analysis of global and regional left ventricular function: a problem revisited. $\mathcal{f} \mathrm{Am}$ Soc Echocardiogr 1990;3:478-87.

22 Schiller NB, Shah PM, Crawford M, et al. Recommendations for quantitation of the left ventricle by twodimensional echocardiography. American Society of Echocardiography Committee on Standards, Subcommittee on Qardiography Committee on Standards, Subcommittee on
Soc Echocardiogr 1989;2:358-67.

23 Stoddard MF, Pearson AC, Kern MJ, et al. Left ventricular diastolic function: comparison of pulsed Doppler echocardiographic and hemodynamic indexes in subjects with and without coronary artery disease. $7 \mathrm{Am}$ Coll Cardiol 1989;13:327-36. 
24 Rinaldi CA, Linka AZ, Masani ND, et al. Randomized, double-blind crossover study to investigate the effects of amlodipine and isosorbide mononitrate on the time course and severity of exercise-induced myocardial stunning. Circulation 1998:98:749-56.

25 Bland JM, Altman DG. Statistical methods for assessing agreement between two methods of clinical measurement Lancet 1986;i:307-10

26 Calnon DA, Glover DK, Beller GA, et al. Effects of dobutamine stress on myocardial blood flow, $99 \mathrm{mTc}$ sestamibi uptake, and systolic wall thickening in the presence of coronary artery stenoses: implications for dobutamine stress testing. Circulation 1997;96: 2353-60.

27 Masani ND, Jones E, Hall RJC. Exercise induced myocardial stunning: differential rates of systolic and diastolic recovery. Br Heart f 1994;71:78.

28 Shaheen J, Luria D, Klutstein MW, et al. Diagnostic value of 12 -lead electrocardiogram during dobutamine echocardio12-lead electrocardiogram during dobutamine

29 Mazeika PK, Nadazdin A, Oakley CM. Dobutamine stress echocardiography for detection and assessment of coronary echocardiography for detection and assessment of co

30 Daoud EG, Pitt A, Armstrong WF. Electrocardiographic response during dobutamine stress echocardiography. $\mathrm{Am}$ Heart f 1995;129:672-7.
31 Mairesse GH, Vanoverschelde JL, Robert A, et al. Pathophysiologic mechanisms underlying dobutamineand exercise-induced wall motion abnormalities. Am Heart F 1998 ; 136 :63-70.

32 Rallidis L, Cokkinos P, Tousoulis D, et al. Comparison of dobutamine and treadmill exercise echocardiography in inducing ischemia in patients with coronary artery disease. f Am Coll Cardiol 1997;30:1660-8.

33 Allen J, Cox TA, Kloner RA. Myocardial stunning following exercise testing in man: early time course. Circulation 1995; 92:551-1.

34 Rinaldi CA, Linka AZ, Masani ND, et al. Systolic functional recovery during exercise-induced myocardial stunning is biphasic. Eur Heart F 1996;17:567-7.

35 Masani ND, Avery PG, Jones E, et al. Perfusion-contraction mismatch after exercise-induced ischaemia: evidence of myocardial stunning in patients with angina. Circulation myocardial stunning in patients with angina. Circulation

36 Selwyn AP, Allan RM, L'Abbate A, et al. Relation between regional myocardial uptake of rubidium- 82 and perfusion: absolute reduction of cation uptake in ischemia. Am 7 Cardiol 1982;50:112-21.

37 Fragasso G, Benti R, Sciammarella M, et al. Symptomlimited exercise testing causes sustained diastolic dysfunction in patients with coronary disease and low effort tolerance. F Am Coll Cardiol 1991;17:1251-5.

\section{IMAGES IN CARDIOLOGY}

\section{Abnormal ventricular conduction following dothiepin overdose simulating acute myocardial infarction}

A 41 year old man was admitted following an overdose of $40,25 \mathrm{mg}$ pills of dothiepin. He was drowsy but otherwise well. ECG on admission showed only minimal broadening of the QRS consistent with a tricyclic overdose, but symmetrical $\mathrm{T}$ wave repolarisation abnormalities appeared within nine hours mimicking an acute anteroseptal myocardial infarction. Serial cardiac enzyme estimates (creatine kinase and aspartate transaminase) did not confirm an ischaemic event. Cross sectional echocardiography was normal with no evidence of focal hypokinesis. ECG changes persisted for six weeks. An exercise tolerance test following the Bruce protocol was normal. Coronary angiography was performed three months later after the patient complained of exertional chest pain. This was also normal.

Such changes in ventricular repolarisation are rare but are a reminder of the electrical dangers inherent in tricyclic use. The changes in conduction were likely to be due either to the quinidine-like activity of dothiepin, or to an alteration in membrane permeability allowing differences in potassium concentrations between different areas of the myocardium, rather than to any ischaemic damage to the myocardium.

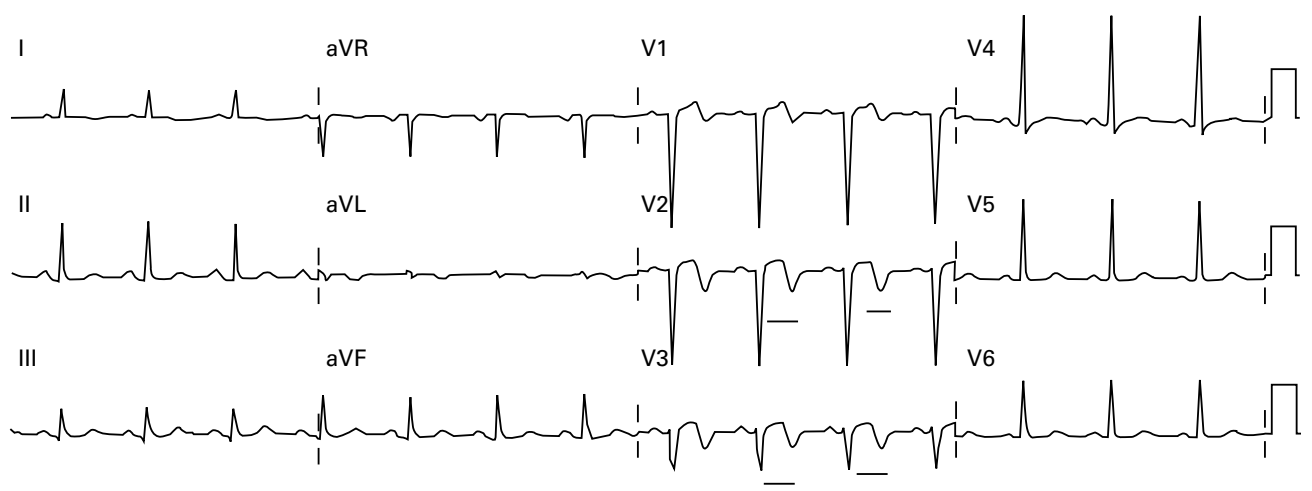

